\title{
Observing Professionals Taking Notes on Screen
}

\author{
Mark Melenhorst \\ University of Twente \\ m.s.melenhorst@utwente.nl
}

\begin{abstract}
In this study 38 participants wrote a piece of advice based on reading and annotating information from an extensive website. Half of the participants took notes in a separate window, the other half used an advanced annotation tool. Intext annotations were far more used than separate notes. The frequency with which features of the note-taking tool notes was used depends on the phase in the process. The association between process phase and the use of features is less clear for the annotation tool. Requirements are formulated for the design of annotation tools.
\end{abstract}

Keywords: annotation, note-taking, usability, writing-from-sources, information processing,

\section{Annotations and Note-Taking Online}

When entering an office, one would probably see papers spread over the desks, which are enriched with notes in the margins, highlights, or other annotations. However, reading texts and annotating them on screen in a similar manner as on paper is far less common.

One of the reasons for the absence of annotations on screen is the ease with which notes can be taken. Annotating (that is: making markings or notes on the texts themselves) on paper is part of the reading process, while on screen it is a separate activity, in a separate part of the interface. For instance, marking a passage requires users to select it, and to find and press the button that marks the passage, while a single stripe suffices for paper ([2]; [3]). Note-taking (making notes in a separate document or on a sheet of paper) is even harder to integrate with reading as another window has to be activated, removing the texts the notes refer to out of view. This attention shift from reading to annotating or note-taking is problematic for readers [3].

The second complicating factor in reading and annotating documents on screen are the spatial conditions. People tend to combine information from different documents by making annotations with both documents lying on their desk simultaneously. This function of annotations is hard to achieve on screen ([1])

Thus, the spatial conditions as well as the attention shift from reading to annotating hinders readers to annotate text on screen. For that reason, developers of annotation software projects seek to emulate the flexibility with which annotations can be taken on paper (for a review see Wolfe, 2002). The focus of these projects is the development of a user-friendly tool. However, the actual use of the annotation tool as part of everyday professional tasks is not addressed by such projects.

Understanding the process of annotation as part of everyday professional tasks is key to develop software that seeks to support reading from screen. One of the tasks in which reading and annotating plays an important role, is the 'writing from sources' task, such as writing a piece of advice based on reports or statistics. This task consists of reading and understanding source texts and transforming the contents in such a way that it suits the reader's needs [6]. Thus, based on the rhetorical situation readers select relevant information from the sources, using that information after adaptation to their own goals to substantiate their writings.

With that purpose in mind, we set up an experiment to answer the following questions: 
1) To which degree and for which purpose are the features of the annotation and note-taking tool used in a writing-from-sources process?

2) How is annotating and note-taking distributed over time in a writing-from-sources process?

Based on the answers to these questions, we can formulate tentative requirements for annotation tools.

\section{Methodology}

Thirty eight employees of the Dutch provinces with expertise on landscape planning were asked to write a piece of advice based on a website with policy documents, and facts and figures.

A $2 \times 2$ design was used. Twenty participants were provided with an advanced annotation tool during the process (the annotation condition), the other participants could use a basic word processor to take notes (the note-taking condition). In both conditions, for half of the participants keywords reflecting core aspects of the tasks were already inserted in the note-taking or annotation tool (the prestructured condition), while this was not the case for the other half of the participants (the unstructured condition).

In the annotation condition, iMarkup (http://www.imarkup.com) was provided to the participants as an example of an advanced annotation tool. The main functions of this tool are displayed in Figure 1: highlighting passages and putting sticky notes on the documents. Both the sticky notes and the highlights can be assigned to self-defined categories. Users can use an overview of the highlights and stickies on the left side of the screen, enabling them to jump to these notes in

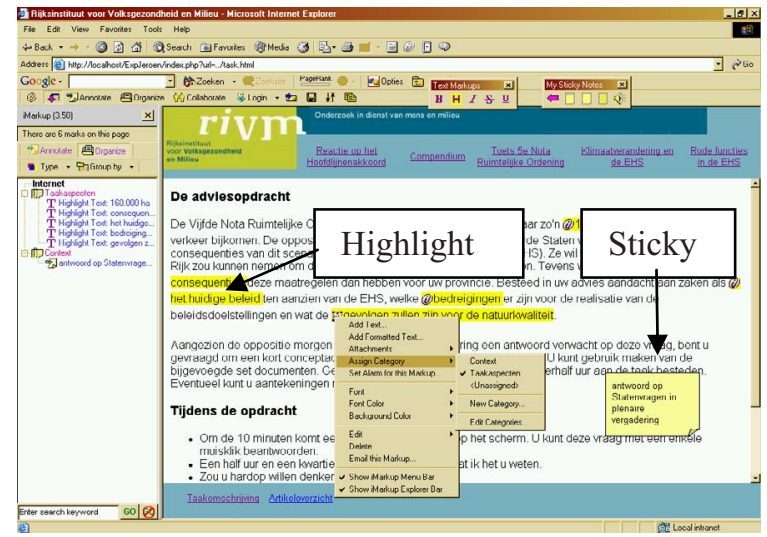

Figure 1 Functions of the annotation tool

their original context by clicking on its title in the overview.

Before the advice task was given, the annotation or note-taking tool was demonstrated. Participants could spend 90 minutes on the advice task itself. Beforehand, the users were given the opportunity to explore the available materials. This phase is referred to as the 'Exploratory phase'.

\section{Results}

In this section we will describe the results of the experiment outlined above.

\section{Use of the tool's features}

We investigated whether tool or task structure influenced the number of instances in which notes were taken. An ANOVA was conducted with tool and structure as independent variables and number of annotation instances as dependent variable. A main effect of tool was found $(\mathrm{F}(1,36)=23.36$, $\mathrm{p}=.000$ ): participants in the annotation condition took significantly more annotations than participants in the note-taking condition. 
Table 1. Annotation frequencies distributed over functionality and process phase

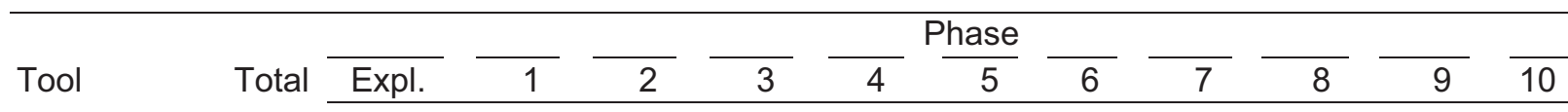

\begin{tabular}{|c|c|c|c|c|c|c|c|c|c|c|c|c|}
\hline \multirow{2}{*}{\multicolumn{13}{|c|}{ Note-taking }} \\
\hline Copied & & & & & & & & & & & & \\
\hline Citation & \multicolumn{2}{|c|}{50} & 8 & 7 & 8 & 14 & 8 & 3 & 1 & 1 & & \\
\hline \multicolumn{13}{|l|}{ Copied } \\
\hline $\begin{array}{l}\text { Citation: } \\
\text { essay }\end{array}$ & \multicolumn{2}{|l|}{47} & 8 & 5 & 5 & 9 & 4 & 5 & 5 & 4 & 1 & 1 \\
\hline \multirow[t]{2}{*}{ Self-written } & 67 & 3 & 33 & 9 & 7 & 6 & 1 & 2 & 3 & 2 & 1 & \\
\hline & 164 & 3 & 49 & 21 & 20 & 29 & 13 & 10 & 9 & 7 & 2 & 1 \\
\hline
\end{tabular}

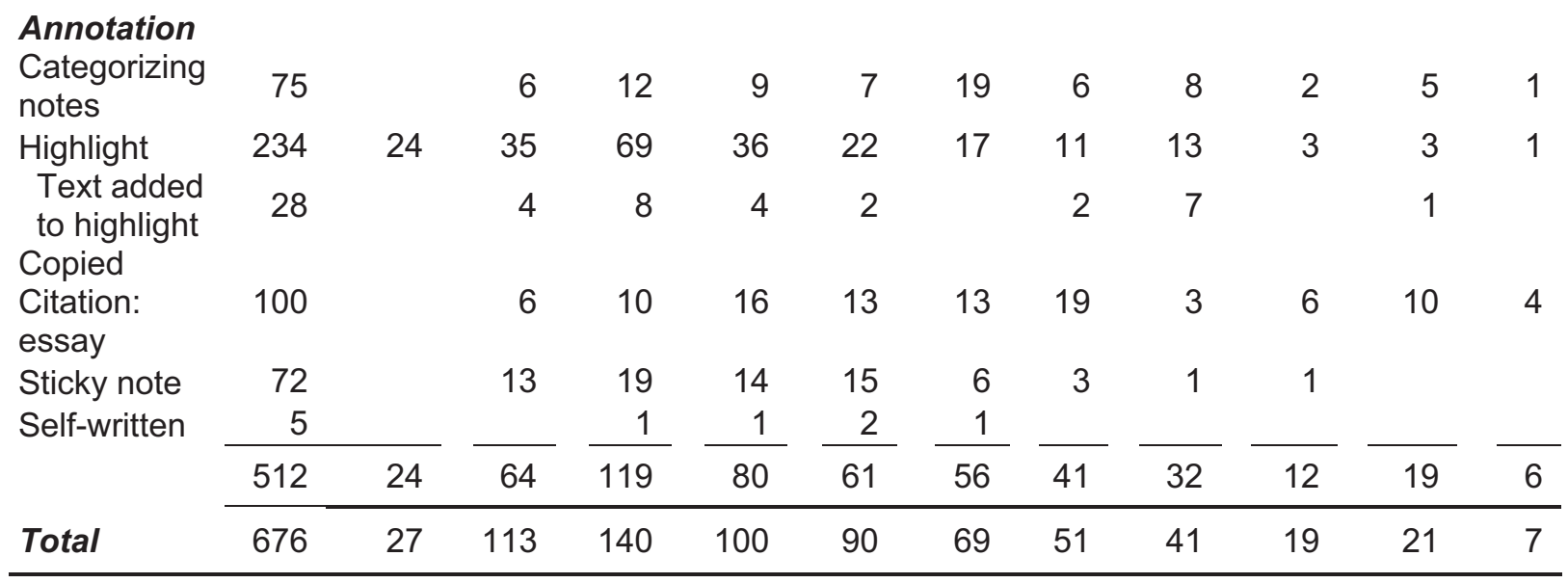

Intuitively, appreciation for and unfamiliarity with a new tool such as iMarkup would explain this effect. The results from the questionnaire provide some evidence for this explanation. On a five points appreciation scale with four items participants in the annotation condition were more positive about the tool $(\mathrm{M}=12,76, \mathrm{SD}=2,28)$ than participants in the note-taking condition $(\mathrm{M}=$ $10,72, \mathrm{SD}=2,16),(\mathrm{t}(33)=-2,72, \mathrm{p}=0,01)$. A more likely explanation, however, is that notes have to be taken in a separate window, while in the annotation condition notes can be taken in the same window. Thus, in terms of O'Hara et al. [3], the attention shift between reading and note-taking is probably considerably larger for the note-taking condition than for the annotation condition.

Confirming this suggestion, Participant 13 using a note-taking tool said "This is not handy. I have to switch back and forth all the time". Thus, the interface seems to have readers use the note-taking tool less than the annotation tool.

In Table 1 the frequencies are displayed with which the different features in the tools were used. We divided the process into 10 phases, each representing $10 \%$ of the process length. The
'Expl.'-phase is the phase in which participants could explore the materials and their contents prior to the advice task.

It can be seen from Table 1 that in the annotation condition, highlights were used most frequently by the users. They had the function of making rereading passages easier. Additionally, by highlighting passages in the assignment participants were able to assess what was expected from them: highlighting helped them construct a task representation [1].

Few remarks have been added to the highlights (28 added remarks on 234 highlights). Apart from that users probably just want to draw attention to relevant parts with highlights only, the number of additional actions required to add comments probably also accounts for this result: users have to select a passage first, click the highlight button, followed by doubleclicking an icon next to the highlight. This sequence of actions may have been too much for the participants, resulting in few added comments to the highlights.

The use of the sticky notes was analyzed with respect to their function in the process. The most 
important function of sticky notes was to support paraphrasing information read. This helped the readers to reread the information. The second function of the sticky notes was to record preliminary ideas for the piece of advice. In terms of McGinley [4], they had the function of an intermediate text between the sources and the eventual piece of advice. When participants used sticky notes in such a manner, it primarily contained an outline for the piece of advice or the topics that had to be dealt with. Some evidence for this function of sticky notes can be found in the annotation frequencies and the protocols. After the initial phases in which ideas are formed, the number of sticky notes declines more rapidly than this is the case for the other features. Participant 19 using iMarkup said while writing a sticky note "what I want to point out, is what should be part of the piece of advice, what will be problematic".

Readers using the note-taking tool copy citations from the sources to the notepad and to the piece of advice. When they write their own notes, they primarily paraphrase information read. While reading the task description in the first phase of the process, readers write the majority of the selfwritten notes.

Participants could copy passages to their piece of advice in both conditions. Strikingly, participants in the annotation condition $(\mathrm{M}=4,75 ; \mathrm{SD}=3,51)$ copied more passages from the sources to their piece of advice than participants in the note-taking condition $(\mathrm{M}=1,67 ; \mathrm{SD}=, 217),(\mathrm{t}(24)=-3,03$; $\mathrm{p}=.006$ ). They may have had the impression that they could only take notes using the tool provided, or they may not have known that it was possible to copy citations to the piece of advice.

Alternatively, using highlights and/or sticky notes may have triggered them to integrate information from the different sources into a coherent representation, reducing the need to draw on the formulations in the texts themselves.

\section{Use of the tool throughout the process}

As can be seen from Table 1, users neither take the same number of notes in each phase of the process nor do they make use of every type of notes to the same extent $(\chi 2(70, \mathrm{~N}=90)=170,12 ; \mathrm{p}=.000)$. Participants take the largest number of notes in the first phases of the process, which is not surprising since reading dominates in the first phases, while writing is the most important activity in later phases.
The use of the tool declined more rapidly over time in the note-taking condition than in the annotation condition. Participants were likely to use the window in which the piece of advice had to be written as an alternative notepad. It may be that once participants started to write their piece of advice, they may have considered the functionality of the separate notepad as partly redundant.

To investigate the strength of the associations between phases in the process and the features of both tools, a correspondence analysis was conducted. In Figure 2 the outcomes of this exploratory analysis are displayed. The closer the points are to each other, the stronger they are associated.

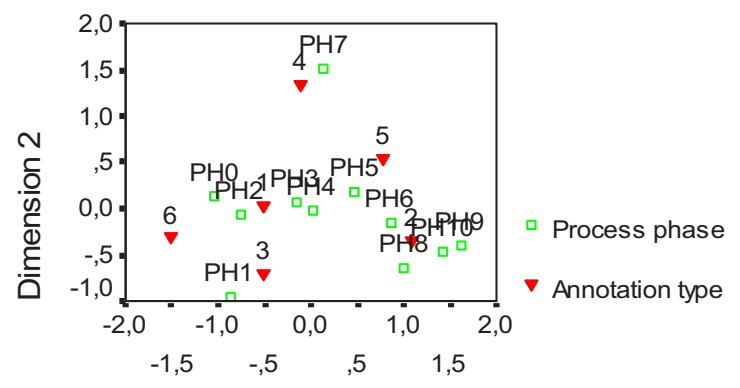

Dimension 1

Note: Annotation type No's refer to:

$$
\begin{array}{ll}
1=\text { Highlight } & 8=\text { Adding text to highlight } \\
\begin{array}{l}
4=\text { Copy passage to } \\
\text { piece of advice }
\end{array} & 9=\text { Assigning a category to } \\
6=\text { Sticky note } &
\end{array}
$$

Figure 2 Correspondence analysis Annotation

No clear patterns emerge with an overlap between phases and features, implying moderate or weak associations. However, it can be seen that highlighting passages (type 1) is associated most to the second, third and fourth phase of the process. Copying passages to the piece of advice (type 2) is associated primarily with the last three phases in the process.

In Figure 3 the correspondence analysis for the note-taking tool is displayed. Three groups of features and phases emerged from the analysis, indicated by the ellipses. As can be seen from the figure, copying passages to the notepad (type 0 ) is related to the second to fifth phase of the process, while copying passages directly to the piece of advice is more related to the last phases of the 
process. The relationship between self-written notes (type 3 ) and the first process phase is less strong. Evidently, the exploration phase in which only on one occasion a note is taken, is unrelated to other types and process phases.

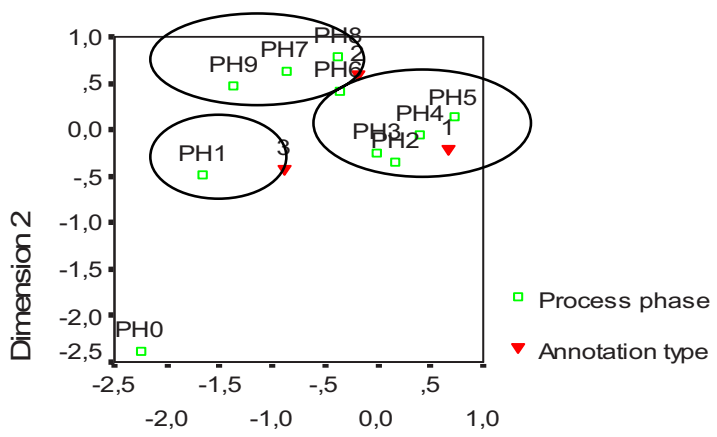

Dimension 1

Note: Annotation type numbers refer to:

\begin{tabular}{|l|l|l|}
\hline $\begin{array}{l}\text { 1= Copying passages } \\
\text { to Notepad }\end{array}$ & $\begin{array}{l}2=\text { Copying passages } \\
\text { to the piece of advice }\end{array}$ & $\begin{array}{l}3=\text { writing own } \\
\text { notes }\end{array}$ \\
\hline
\end{tabular}

Figure 3 Correspondence analysis - Note taking

\section{Conclusions}

Based on the results we can draw conclusions with respect to the use and functions of the annotation and note taking tools and their distribution over time. The annotation tool is primarily used to facilitate rereading. The sticky notes are used to plan the writing process. The features are not clearly associated to process phases.

The note-taking tool is used far less frequently, probably because it is presented in a separate window. In early phases of the process, readers write their own notes. Later, they copy citations to Notepad and directly to the piece of advice. Thus, it seems that note-taking results in a more systematic reading - note-taking - writing process than annotation.

Based on the results that were described in the previous section, and previous research we could formulate tentative recommendations to designers of annotation tools. Integrating the use of the tool in such a complex task as writing-from-sources without using paper, appeared to be troublesome. Consequently, in designing annotation tools the use of the features should be immediately clear to the users as well as how the new features relate to practices in the paper world. Thus, learnability is the key usability principle [5]
The remarks of the participants show that shifting back and forth from one window to the other is complicated, resulting in a problematic transfer from annotations and worthy citations to the piece of advice in the annotation condition and an only meagerly use of the tool in the Notepad condition. An environment in which the reader can both modify the piece of advice and read and annotate the source documents with an easy transfer of information from source to piece of advice may overcome this 'attention shift' problem.

In spite of the chances for software developers described above, the flexibility and easy manipulability of paper will remain an important argument to continue printing documents and making paper annotations instead of reading and annotating on screen.

\section{References}

[1] L. Flower. "The Role of Task Representation in Reading to Write" in Reading to Write. Exploring a Cognitive \& Social Process. L. Flower et al, Eds. New York: Oxford University Press, 1990, pp. 3575

[2] K.P. O'Hara and A. Sellen. "A Comparison of Reading Paper and On-Line Documents". Proc. of the SIGCHI Conf. On Human Factors in Computing Systems, 1997, pp. 335-342

[3] K. P. O'Hara, A. Taylor, W. Newman, \& A.J. Sellen. "Understanding the materiality of writing from multiple sources". International Journal of Human-Computer Studies, 56, pp. 269-305, 2002

[4] W.J. McGinley "The role of reading and writing while composing from sources" Reading Research Quarterly, 27(3), p. 227-248, 1992

\section{[5] J. Nielsen. Usability Engineering. Boston:} Academic Press, 1990

[6] N.N. Spivey. "Transforming Texts. Constructive Processes in Reading and Writing" Written Communication, 7(2), pp. 256-287, 1990

[7] J. L. Wolfe. "Annotation Technologies. A software and research review" Computers and Composition, 19, pp. 471-497, 2002 


\section{About the Author}

Mark Melenhorst is a $\mathrm{PhD}$ candidate participating in the Technical Communication group of the University of Twente. His research interests include document design, interface and interaction design, and usability testing. 